\title{
OCT-Based Velocimetry for Blood Flow Quantification
}

\author{
Boy Braaf, Maximilian G. O. Gräfe, Néstor Uribe- \\ Patarroyo, Brett E. Bouma, Benjamin J. Vakoc, \\ Johannes F. de Boer, Sabine Donner, \\ and Julian Weichsel
}

\subsection{Introduction}

A complex network of blood vessels within the retina and the choroid ensures the perfusion of the photoreceptors, ganglion cells, retinal nerve fiber bundles and other retinal tissues essential for the visual system. Because various retinal diseases originate from pathologic changes in the local hemodynamics, localized blood flow measurements could potentially serve as a disease biomarker.

Fluorescence angiography is the standard clinical tool for qualitatively visualizing the structure of the blood vessel network in the back of the eye. Early detection of perfusion-related

B. Braaf $\cdot$ N. Uribe-Patarroyo

Wellman Center for Photomedicine, Harvard Medical

School, Massachusetts General Hospital,

Boston, MA, USA

M. G. O. Gräfe · J. F. de Boer

Vrije Universiteit Amsterdam,

$\mathrm{HV}$, Amsterdam, The Netherlands

B. E. Bouma · B. J. Vakoc

Wellman Center for Photomedicine, Harvard Medical School, Massachusetts General Hospital,

Boston, MA, USA

Institute for Medical Engineering and Science,

Massachusetts Institute of Technology,

Cambridge, MA, USA

S. Donner $\cdot$ J. Weichsel $(\bowtie)$

Heidelberg Engineering GmbH,

Heidelberg, Germany

e-mail: Julian.Weichsel@HeidelbergEngineering.com diseases, monitoring disease progression, and evaluating the effectiveness of therapeutic intervention, however, could potentially be improved by robust methods for quantifying blood flow dynamics. Studies have shown the overall clinical potential of quantitative flow measurements in retinal diseases like AMD [1], glaucoma [1-4], diabetic retinopathy $[5,6]$ and others. Consequently, a number of different techniques have been developed for this purpose and the individual strengths and weaknesses of the various approaches have been discussed comprehensively in previously published review articles [1, $2,7]$. Despite progress, it remains difficult to reliably quantify blood velocities within individual blood vessels using these existing techniques. In addition, some methods report rather complex hemodynamic parameters, which are not easily interpreted and often dependent on the specific measurement method employed. Measurement uncertainty and variation have even led to contradicting study results in the published literature for the same disease and under otherwise similar conditions [7]. To date, no gold standard has been established for quantitative ophthalmic blood flow- and velocimetry.

Recently, OCTA became commercially available as a tool for qualitative, non-invasive, 3D visualization of the blood vessel network of the posterior eye. In research studies as well as in the daily clinical routine, OCTA is on the way to becoming an accepted imaging modality for the 
diagnosis of retinal vascular disorders including neovascularization $[8,9]$ and retinal vein occlusion [10]. Current clinical implementations of OCTA provide an almost binary discrimination between static tissue and blood vessels with sufficient flow, and a very limited ability for flow velocity quantification. This makes OCTA especially applicable in clinical scenarios when vessel perfusion drops out completely or when pathologic vasculature is growing into otherwise avascular regions of the retina. Statistical analysis of the OCTA signal mainly focuses on numerically evaluating the qualitative vascular signal and local features in the microvasculature geometry as well as the area of extended ischemic regions [11, 12] and avascular zones. Although these analyses are sometimes termed 'quantitative OCTA' in the literature, they are not based on actual quantitative OCT velocimetry measurements of blood flow. Instead the outcome of different established OCTA signal reconstruction algorithms is used in the analysis. As the visibility and detectability of blood flow can be highly dependent on the sensitivity of the specific OCTA algorithm [13, 14], these measurements are again not easily comparable between different devices. In order to arrive at a consistent uniform metric for quantitative blood flow measurement in the future, a robust, easy to use, and reliable tool for clinical flow quantification in the eye is in demand. OCT velocimetry holds the potential to meet these requirements using non-invasive measurements. The principle of OCT-based flow velocity measurements is straightforward; an incident OCT beam is scattered from moving erythrocytes within retinal vessels (Fig. 7.1) and by carefully controlling the spatial and temporal sampling of repeated measurements the underlying blood velocity can be derived from the modulation of the back-scattered signal.

This chapter describes the current status of quantitative OCT-based velocimetry methods for blood flow quantification. It builds upon the description of OCT and OCTA signal processing in Chaps. 3 and 6, respectively. In the first part of this chapter, the motivation and potential for OCT-based retinal flow measurements in clinical

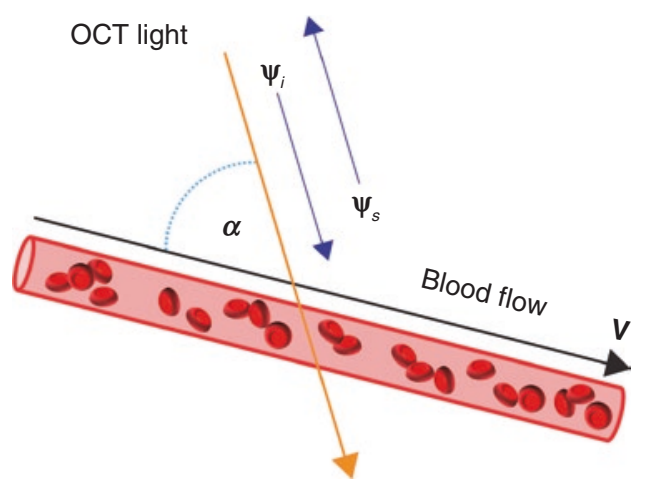

Fig. 7.1 Schematic drawing of an OCT measurement on a blood vessel. The Doppler angle $\boldsymbol{\alpha}$ is defined between the incoming OCT light beam and the direction of the flow velocity $\boldsymbol{V}$. The incident light wave $\boldsymbol{\Psi} i$ is partially reflected by the moving red blood cells into the scattered light wave $\boldsymbol{\Psi}_{s}$. The blood cell velocity is derived by examining the modulation of the scattered light wave from repeated measurements in time. The figure is adopted from Braaf [15]

research and practice is discussed. The subsequent part of the chapter summarizes different methods for flow quantification. These methods are organized by the component of the scattered signal that is analyzed: phase-based methods, amplitude-based methods, and complex-signal based methods that simultaneously use both phase and amplitude.

\subsection{Clinical Potential for OCT- Based Retinal Blood Flow Measurements}

Measurements of retinal blood flow have been employed in ophthalmic clinical research over the last several decades in order to characterize hemodynamic regulation in healthy eyes and to assess its implications in eyes with retinal conditions [16]. A diverse toolkit of pioneering technical approaches has been used in these studies [7], including scanning laser Doppler flowmetry $[17,18]$ and tracing dye concentration dynamics over time with fluorescence angiography [19]. However, despite this tremendous investigative effort, no truly quantitative measurement of physical blood velocity or flow rate has yet made a successful and permanent transition into 
clinical practice. Instead, only qualitative hemodynamic features are routinely assessed [20].

To some extent, the hesitation towards clinical acceptance can be explained by limited usability of some modalities, which inevitably diminishes measurement precision and accuracy in the busy clinic. Additionally, some quantification techniques yield rather complex or only relative metrics that are mere proxies for the desired physical observables velocity (distance per time) and volumetric flow rate (volume per time). The interpretation of current measurements and the direct comparison between different methods is therefore difficult, especially considering the complexity of physiologic hemodynamics in the eye. Blood flow is auto-regulated in combination with several other factors such as blood pressure and tissue oxygenation, while it also varies depending on retinal location and over time. Therefore, disentangling the influence of all involved factors requires reliable, comparable, and localized flow measurements. Due to this complexity and the lack of accurate measurement tools many of these factors have not been systematically assessed in clinical research studies so far. Consequently, this has led to a significant portion of conflicting study results about the influence of hemodynamics on pathologies such as diabetic retinopathy [16] and glaucoma [2, 4].

Since its commercial introduction into clinical ophthalmology in 2014, OCTA imaging has had an impressive impact on research and daily clinical disease management (cf. Chap. 6). This is certainly due to its capability to noninvasively reveal the three dimensional (3D) volumetric structure of the retinal vascular network, while the measurement itself is relatively easily accessible, fast, and robust. However, to some extent the success of OCTA may also seem surprising as the interpretation of its resulting signal is not without problems. These issues include the impact of artefacts and also the qualitative signal characteristics of this technology. Without exception, every current commercially available implementation of clinical OCTA aims at reliably discriminating locations of motion from static tissue within the OCT volume scan. However, the OCTA signal offers essentially no resolution with respect to blood velocity. Therefore, current OCTA imaging is almost blind to subtle changes in blood flow that occur either entirely above or below the sensitivity threshold of the angiographic signal. This becomes quite obvious for instance, when considering the almost vanishing impact of the cardiac cycle on extended OCTA volumes. Several periods of diastole and systole are encoded into the sequentially acquired OCTA scans, but typically these variations cannot be differentiated in the resulting angiographic signal. The specific sensitivity threshold for the quasi-binary signal reconstruction task (i.e. dynamic vs. static), however, depends on the angiographic signal reconstruction algorithm employed and differs amongst the commercially available devices. Thus, regions of rather slow motion may be detected in one, while remaining unnoticed in another method, depending on their individual sensitivities to motion [21]. Nevertheless, there is increasing scientific and clinical interest in deriving statistical parameters, like vessel density and geometric features of capillary networks, from such qualitative OCTA datasets and relating these metrics to retinal pathologies. Due to the mentioned differences in the available devices and OCTA signal reconstruction techniques, such analyses will have to be cautiously interpreted in order to not produce apparently conflicting study results. Otherwise such results may, at least to some extent, undermine the emerging clinical acceptance and trust in this new imaging modality in the future.

In light of these apparent current limitations, supplementing qualitative OCTA imaging with additional quantitative OCT-based measurements in order to directly and reliably determine blood flow velocity and flow rate in absolute physical units seems a logical next step. Although it is unlikely that currently available clinical OCT hardware is capable of providing quantitative velocity measurements with the same field of view as qualitative OCTA implementations, localized quantification of blood velocity in a robust and easy-to-use OCT-based measurement has the potential to further support the interpretation of OCTA data. Such measurements could offer the potential to improve inter-device 
reproducibility, overall sensitivity, and the dynamic range of the OCTA signal. For true velocity measurements, the accuracy and precision of different devices could be directly assessed using independent validation techniques in vivo that may be more invasive or technically challenging, but can serve as an accepted goldstandard for initial validation [22, 23]. Given the additional ability of OCTA to accurately determine vascular geometry in $3 \mathrm{D}$ and therefore also the localized cross-section of individual vessels, the direct conversion of measured velocity to volumetric flow rate is readily available in this technique.

Although it is difficult to confidently predict the future importance of quantitative blood flow measurements in clinical ophthalmology, flow is generally considered to be an important variable in the characterization of the eye in health as well as at the onset and progression of disease:

Healthy eyes-Due to the 3D spatial resolution of OCT, flow measurements could, in principle, be ascribed to individual and distinct retinal capillary plexuses. Such networks have been defined histologically and can also be identified and discriminated in OCTA data. Even in healthy eyes, however, a large variability of blood velocity and flow measurements in the retina has been reported. This is partly due to the different technical methods that have been used for deriving the measurements, but also to contributions from confounding factors such as intra-ocular pressure, blood pressure, and the amount of oxygen supply. This variance makes a meaningful characterization of hemodynamics challenging and suggests that robust velocity measurements will require new instrumentation and possibly methods for controlling for extraneous physiological variation.

Glaucoma-Although a reduction of retinal blood flow in glaucoma patients has been observed, the precise role of vascular disturbances as a cause or result of the disease remains controversial [3, 4]. However, as intraocular pressure (IOP) is currently the only treatable risk factor in glaucoma, the potential for interventions in blood flow as an additional therapeutic option in the future is certainly compelling. Additionally, in some scenarios vascular irregularities may precede structural changes in the eye and could be used as a marker for early diagnosis and progression monitoring. These hypotheses are especially plausible in progressing cases of primary open angle glaucoma, where intra-ocular pressure has already been lowered therapeutically, or in normal tension glaucoma. Here, the disease state may be progressing despite having IOP in the normal range of $12-22 \mathrm{mmHg}$, which currently leaves no further viable options for therapeutic intervention.

Diabetic retinopathy (DR)_Earlier studies reported both increased as well as decreased retinal blood flow in early DR [5]. Since the clinical admission of OCTA devices, ischemic regions have been quantified and characterized based on their resulting OCTA signal [12]. As discussed above, the introduction of OCT-based quantitative physical flow measurements will ultimately help improve the device independent reproducibility of the derived parameters for ischemia. Additionally, the improved sensitivity and greater dynamic range holds the potential to reliably detect even subtle changes at the onset of the retinal disease.

Age related macular degeneration (AMD) - The observation of flow alterations in dye-based fluorescence angiography, especially in the choriocapillaris and choroid, has been associated with AMD [1]. As the choriocapillaris provides metabolic support for the retinal pigment epithelium (RPE), such alterations in blood supply could explain some of the observed pathologic manifestations of AMD such as drusen in its nonexudative form, as well as the development of choroidal neovascularization (CNV) in exudative AMD. Both the choriocapillaris and the choroid are located below the highly reflective RPE. Therefore, using OCT it will be more challenging to collect sufficient signal strength for reliably reconstructing blood velocity from below an intact RPE compared to the vascular networks located in the inner retina.

Provided that accurate and precise flow measurements can be achieved with OCT, we anticipate that this new capability has the potential to become a very useful tool that can characterize retinal hemo- 
dynamics and derive associated biomarkers for various diseases. Along that path, this new clinical technique could help to resolve apparent conflicting results that emerged in previous studies using other measurement approaches. Additional potential lies in combining quantitative flow measurements with oximetry data in order to build a comprehensive and coherent understanding of the hemodynamic regulatory system in the future.

\subsection{Measuring Blood Flow with OCT}

\subsubsection{Phase-Based Methods}

In this section, we discuss OCT flow quantification methods that are based on the detection of signal phase or frequency changes that occur between successive OCT measurements on moving scattering particles. These methods are published in the literature under a variety of names including 'Doppler OCT', 'phase-resolved OCT' and 'joint spectral and time domain OCT'.

\subsubsection{Theory}

In OCT, frequency changes from axial scattering particle motion, parallel or antiparallel to the propagation direction of the illumination light, are observed within the interference term of the detector signal. Under the simplifying assumption of a single scattering particle, reflecting light at the axial position $z_{s}$ in the OCT interferometer sample arm and a fixed interferometer reference arm length $z_{R}$, the resulting interference signal at the detector will show the dependency:

$$
I_{\text {det }}(k) \propto \cos (\underbrace{2\left(z_{S}-z_{R}\right)}_{\omega_{s}} k) .
$$

Hence, the oscillatory intensity signal $I_{d e t}(\mathrm{k})$ is a function of the wavenumber $k$ with modulation frequency $\omega_{s}$. Axial motion of a scattering particle will change $z_{s}$ and therefore $\omega_{s}$ in repeated acquisitions, accordingly. This change in the modulation frequency due to axial motion can be observed in and extracted from the OCT signal [24].
Standard practice in Fourier-domain OCT (FD-OCT) processing is the transformation of the $k$-dependent intensity signal into a depth dependent $\left(z_{s}\right.$-dependent $)$ complex signal via Fourier transformation. The (small) change in the axial position of moving scattering particles changes $\omega_{s}$ in Eq. (1) and manifests in a phase change in the transformed complex signal from sequential measurements [25]:

$$
\Delta \varphi=\frac{4 \pi n \tau V \cos \alpha}{\lambda_{0}}
$$

with $n$ as the refractive index of the medium, $\tau$ as the time difference between repeated measurements, $V$ as the scattering particle velocity, $\lambda_{0}$ as the central wavelength of the OCT light source and Doppler angle $\alpha$ as the angle between the light propagation and the flow velocity directions as illustrated in Fig. 7.1. The Doppler frequency shift associated with the particle motion can be calculated from the phase change $\Delta \varphi$ of Eq. (2) as:

$$
f_{D}=\frac{\Delta \varphi}{2 \pi \tau}=\frac{2 n V \cos \alpha}{\lambda_{0}} .
$$

Equations (2) and (3) indicate that the Doppler frequency shift is proportional to the projected axial component of the velocity $v_{z}$ along the incident beam direction, $v_{z}=V \cos \alpha$.Consequently, only when the Doppler angle $\alpha$ is known, the actual flow velocity $V$ be calculated [26].

The improvement in acquisition speed introduced by FD-OCT enabled the in vivo volumetric measurement of axial velocities in the retina [2730]. The OCT phase change $\Delta \varphi$ measured from within individual blood vessels as shown in Fig. 7.2a visualizes both the direction and the magnitude of the axial velocity component of blood flow that is either parallel or antiparallel to the incident OCT beam. In addition the dynamics of the cardiac cycle in arteries and veins can be observed (Fig. 7.2b).

The dynamic range of this measurement is defined as the ratio of the maximum and minimum detectable signal. In Doppler OCT the maximum signal is given by the velocity at which the phase 
a

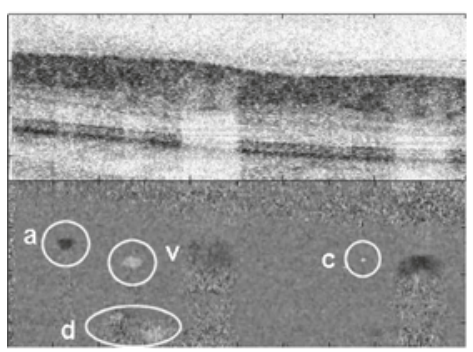

Fig. 7.2 (a) Single frame from a movie showing the repeated acquisition of structural (top) and bi-directional axial blood flow (bottom) B-scans in the retina. Delineated regions in the flow image indicate: a-artery, v-vein, c-capillary, d-choroidal vessel. (b) Integrated flow sig-

difference is still uniquely identified. The phase is cyclic and restricted to the range $[-\pi,+\pi)$ radian. Flow velocities that correspond to larger phase differences outside this range are wrapped back into the original interval and cannot be uniquely identified anymore. Although phase unwrapping algorithms can be used to extend the velocity range, in practice these methods are often difficult to implement in a sufficiently robust way [31, 32]. Without phase unwrapping, the maximum detectable velocity is associated to a phase difference of $\pm \pi$ and given by:

$$
\nu_{z, \max }= \pm \frac{\lambda_{0}}{4 n \tau \cos \alpha} .
$$

The minimum detectable signal is determined by the noise level of the measurement. The overall phase noise has two main contributions: shot noise and positioning error. Shot noise is determined by the optical power in the interferometer reference arm [33]. Regarding positioning error, in order to measure the phase shift or Doppler frequency each sample position has to be measured at least twice. A beam displacement between these two sequential scans causes a variation in the ensemble of illuminated scatterers. This rigid bulk sample displacement, for instance caused by whole sample motion or inaccurate beam positioning, can in general be decomposed b

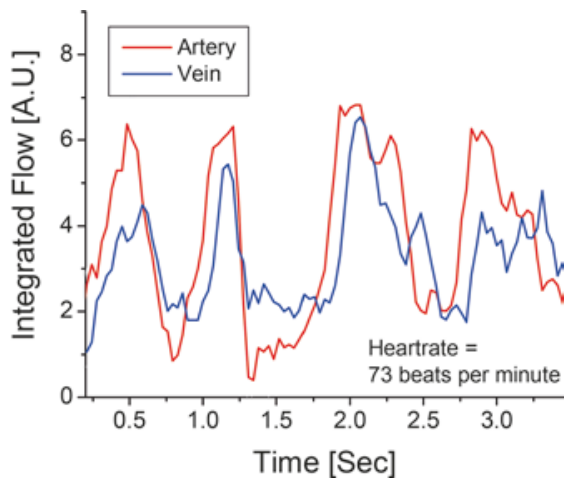

nal over time for an artery and a vein. The dynamics of the cardiac cycle are visible as a temporal pulsation. Reprinted from White et al. [28], with permission of The Optical Society (OSA)

into an axial and a lateral component. Bulk displacement can be compensated in post-processing to some extent only and any remaining positioning error $\Delta x$, relative to the beam spot diameter, leads to an additional contribution to the overall phase noise [28, 33, 34]. Blood flow perpendicular to the beam direction can also be seen as a localized lateral displacement of the measured sample and therefore contributes to or even dominates this noise term for significant lateral flow. The two phase noise contributions are statistically independent and their combined impact on the overall standard deviation $\sigma_{n}$ of the phase shift statistics can be expressed as [33, 34]:

$$
\sigma_{n}=\sqrt{\sigma_{S N R}^{2}+\sigma_{\Delta x}^{2}}
$$

where $\sigma_{S N R}$ describes the shot noise contribution and $\sigma_{\Delta x}$ describes the positioning error contribution to the phase noise respectively.

A schematic illustration of possible Doppler phase shift measurements for different (axial) flow velocities is shown in Fig. 7.3. The signal from static tissue forms the background noise floor from which flow signals are differentiated, and is denoted here as the gray phase noise probability density function $p$ with standard deviation $\sigma_{n}$. For flow signals, $p$ gets phase shifted proportional to the mean axial flow velocity parallel to 


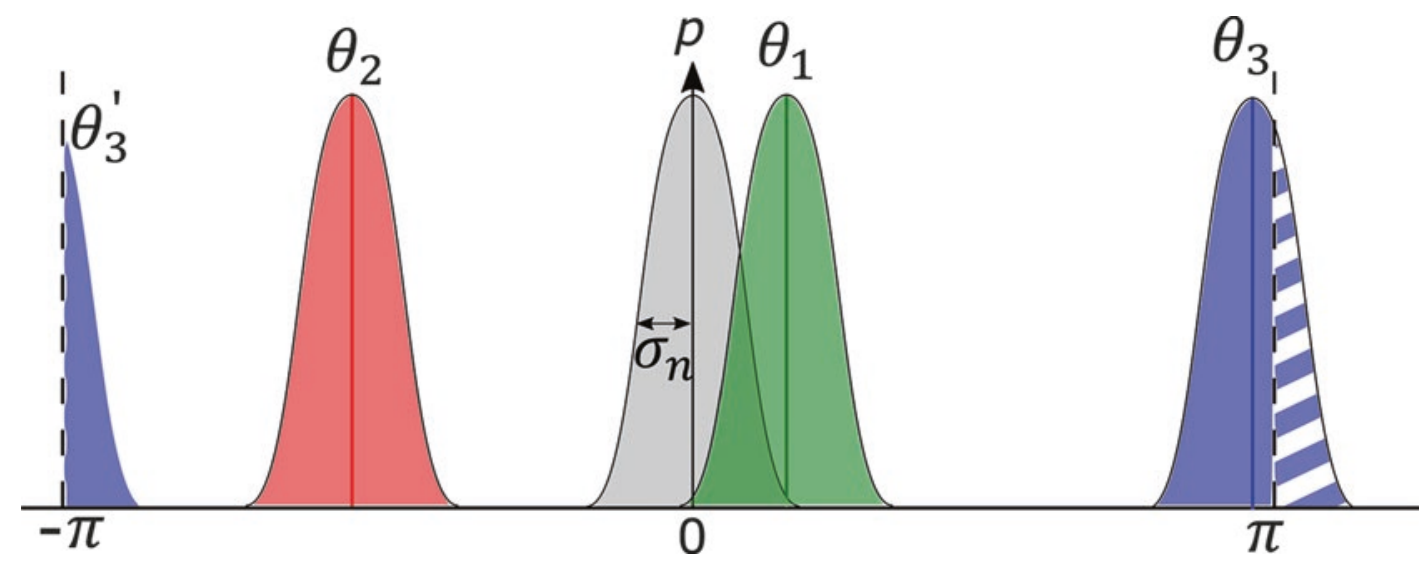

Fig. 7.3 Schematic illustration of the probability density functions for Doppler phase shifts measured for static tissue (gray) and for three different (axial) flow velocities within the phase range $[-\pi,+\pi)$. The static tissue background noise has a probability density function $p$ with

the incident OCT beam (Eq. 2) and its width is further broadened by the lateral flow component perpendicular to the beam. Three possible flow signals are indicated in Fig. 7.3 by distributions centered on an average phase shift $\left(\theta_{j}\right)(j=1,2$, $3)$. A weak flow signal ( $\theta_{1}$ in green) can overlap significantly with the static tissue background noise. In this case the flow signal cannot be easily distinguished from the background. On the other hand, a significantly strong flow $\left(\theta_{2}\right.$ in red) can be completely differentiated from the background noise. As long as this probability density function is still within the $[-\pi,+\pi)$ range, the velocity can be correctly quantified. If the velocity is too high $\left(\theta_{3}\right.$ in blue) the distribution can shift partially or fully outside the $[-\pi,+\pi)$ range. In this case, exceeding phase shift values are wrapped across the periodic interval boundaries and appear on the opposite side of the range $\left(\theta_{3}{ }^{\prime}\right)$. This makes the flow quantification prone to errors, since the flow velocities can appear higher or lower or even change in directionality.

\subsubsection{Application to Retinal Imaging}

As shown in Eq. (2), the unambiguous reconstruction of the velocity of moving scattering particles requires additional knowledge of the direction of their motion as given by the Doppler angle $\alpha$. In the following paragraphs, four quan- standard deviation $\boldsymbol{\sigma}$ n. Flow measurements sampled from distribution $\boldsymbol{\theta}_{\mathbf{1}}$ (green) cannot be easily distinguished from noise, $\boldsymbol{\theta}_{\mathbf{2}}$ (red) can be measured at its true value, while $\boldsymbol{\theta}_{\mathbf{3}}$ (blue) is affected by phase wrapping and therefore prone to errors

titative Doppler flow methods are presented that calculate the true flow velocity from the axial velocity component by either estimating $\alpha$, or by an $\alpha$-independent Doppler signal acquisition and analysis. Afterwards, an alternative Doppler method is discussed that includes a more detailed analysis of the Doppler frequency distribution to separately determine the axial and lateral velocity components. This method might be especially interesting for blood vessels at close to perpendicular orientation to the OCT incident light - a typical scenario in retinal applications - since it is able to measure the lateral flow component.

\section{Circumpapillary Scan}

As OCT is a volumetric imaging modality, under certain conditions it is possible to reconstruct blood vessel orientations (corresponding to the Doppler angle $\alpha$ ) in three spatial dimensions directly from the OCT dataset. This can be done most reliably for large vessels with a significant axial flow component, such that the Doppler signal is clearly above the level of the residual phase noise. These conditions are most reliably met within the peripapillary region where the central retinal artery and vein are located. Wang et al. [35] demonstrated a measurement based on multiple circular scans of the circumpapillary region 

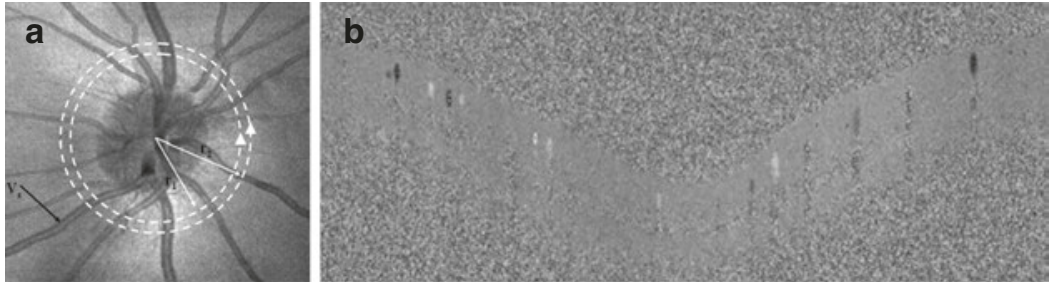

Fig. 7.4 (a) Illustration of the path of two circular scans with different radii around the optic nerve head. (b) Doppler OCT image with grayscale display of the Doppler phase shifts. The image extends from $0^{\circ}$ to $360^{\circ}$ around

at two different radii (Fig. 7.4a). The difference of the radii is chosen small enough that the vessel segments recorded on those circles can be assumed straight across this distance. An example of the measured Doppler phase shifts in one circular scan is illustrated in Fig. 7.4b. Based on their change in axial location between the OCT images obtained at both radii and the difference in the radii of both scans, an estimate of the Doppler angle $\alpha$ can be calculated. Together with the directly measured axial velocity component $v_{z}$, the true velocity can be determined. In addition, if the surface perpendicular to the flow velocity is also calculated, the flow velocities can also be converted into flow rates within the cross sections of the vessels.

\section{En Face Plane Doppler OCT}

Quantitative Doppler flow measurements from cross-sectional OCT scans have the disadvantage that they require the explicit calculation of the Doppler angle. Alternatively, quantitative Doppler flow calculations can be performed on three-dimensional OCT datasets without the need for explicit angle calculations. While the measured axial velocity component is scaled by the cosine of the Doppler angle $\cos \alpha$, the crosssectional area of a blood vessel in an en face image plane (the lateral plane) will scale inversely due to its angled orientation by $1 /|\cos \alpha|$ [36]. These two effects therefore cancel when integration is performed in the en face plane of the three-dimensional OCT dataset and quantitative flow information can therefore be obtained without explicit knowledge on the Doppler angle. the optic nerve head. The dark and bright spots in the retina indicate the flow in opposite directions within the large retinal vessels. Reprinted from Wang et al. [35]

\section{Multiple Beam Doppler OCT}

As large vessels branch out and extend from the optic nerve head through the retina, their diameter becomes smaller and the vessel orientation is harder to extract from OCT measurements. Hence several methods have been developed that do not require vessel orientation, but are able to reveal flow velocities in each individual voxel of an OCT scan directly. A prominent example is the detection and/or illumination with multiple beams, as for instance three-beam illumination [37].

In multi-beam methods a fixed angular separation is used between the illumination and detection beams. This type of beam configuration ensures that one or multiple beams will have a significant Doppler angle with the target blood vessel, and problems with perpendicularly oriented vessels are therefore mitigated. The undilated pupil typically limits the lateral displacement of the illumination and detection beams to $2 \mathrm{~mm}$ on the cornea and consequently restricts the angular beam separation on the retinal surface to be within $\pm 2.4^{\circ}$ from the perpendicular. Although these Doppler angles are relatively small, together with proper calibration, accurate Doppler velocity and flow measurements can be obtained from the axial velocity components and the known angular differences between the beams. The primary disadvantage of this approach is that a complex apparatus is required to generate the multiple beams and this complexity may not be suitable for clinical instruments.

The three-beam illumination method was used to characterize the dependency between vessel diameter and flow velocity for arteries and veins which showed a linear dependency (Fig. 7.5) [37]. It was concluded from this study that the retinal 


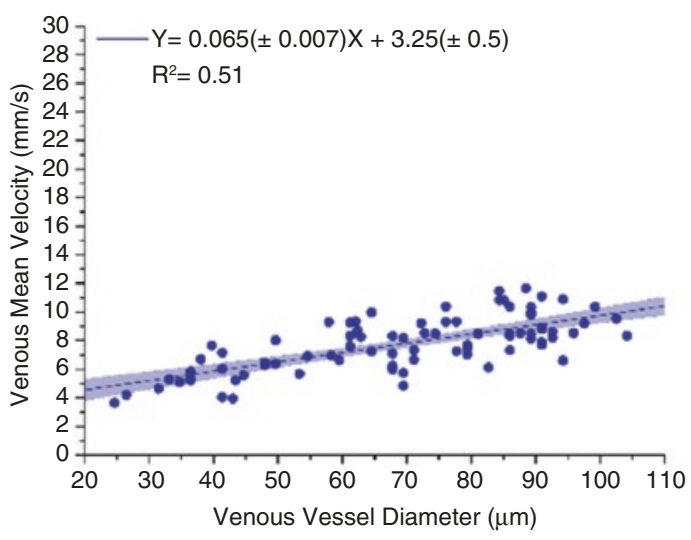

Fig. 7.5 Mean velocity versus vessel diameter as measured with three-beam Doppler OCT. Left: venous velocity. Right: arterial velocity. Filled area: $95 \%$ confidence interval of the fit. The results show a linear relation

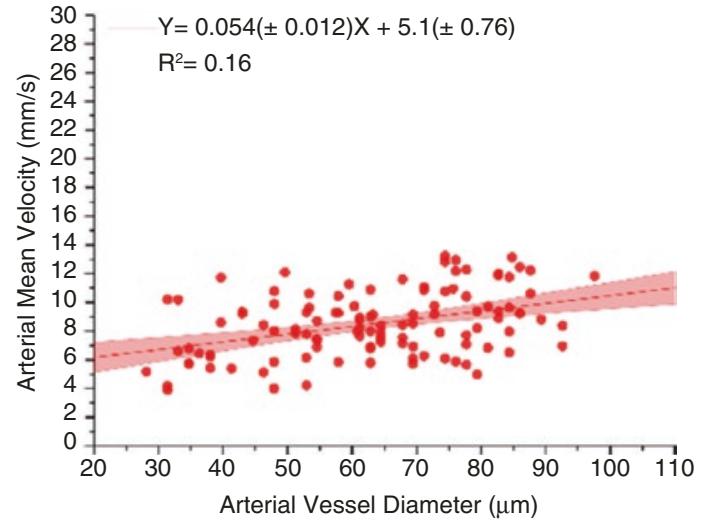

between the vessel diameter and the flow velocity. Reprinted from Haindl et al. [37], with permission of The Optical society (OSA)

the full field of view is captured with a high framerate $2 \mathrm{D}$ camera at a series of wavelengths while slowly sweeping the light source, resulting in an effective A-line rate of about $39 \mathrm{MHz}[39,40]$. The high volume acquisition rate and the phase stability over the full volume are necessary for the successful acquisition of phase stable OCT volumes using FF-SS-OCT. Unfortunately the use of a spatially coherent light source in such instruments greatly increases the detection of multiply scattered light, limiting the usable image depth. In addition, due to the slow wavelength sweep, patient motion that would not affect point scanning clinical systems can degrade the FF-SS-OCT image quality [39, 41, 42].

Nevertheless, an experimental realization of this method was demonstrated for in vivo retinal flow measurements to create velocity maps and for tracking the thermal expansion of excised porcine retinas [40] (Fig. 7.6). Once repeated volumetric scans were acquired, the illumination directions could be chosen freely in postprocessing within the range of acquired wave vectors. As seen in Spahr et al. [43], retinal vessels produce artifacts due to the slow wavelength sweep and multiply scattered photons, which suggests that the applicable velocity range of this technique is limited to a smaller velocity range than the techniques discussed earlier. rapid sweep over the light source bandwidth and data acquisition of one A-line location per sweep, 

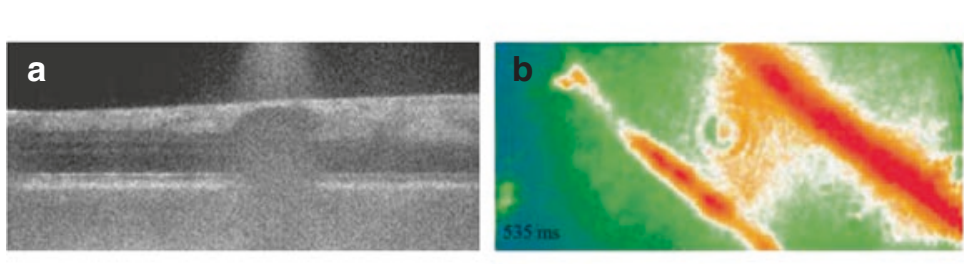

fast

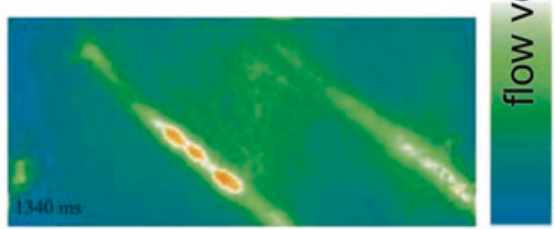

slow

Fig. 7.6 (a) Structural image of ten averaged B-scans from an FF-SS-OCT system. The slow wavelength sweep and multiple scattered photons from large blood vessels create artifacts in the image [43]. (b) The same setup was also used for flow velocity measurements in retinal ves-

\section{Analysis of the Doppler Frequency Bandwidth}

All previously introduced methods for phasebased flow quantification measured a Doppler signal that is for the greater part related to the axial velocity. If the axial velocity is very small due to a nearly perpendicularly oriented vessel, the required measurement accuracy of the Doppler angle $\alpha$ to extract a meaningful flow velocity becomes prohibitive. In the human retina this aspect becomes extraordinarily challenging for blood vessels far away from the optic nerve head that are oriented along the retinal surface. These vessels typically have a Doppler angle that is almost perpendicular to the incident beam direction. To overcome this limitation, a different approach can be employed that utilizes the effect of lateral sample motion to the phase or frequency of the OCT signal.

The OCT phase shift and corresponding Doppler frequency resulting from scattering particle motion are random variables for which the means are given in Eqs. (2) and (3), respectively. Motion, however, not only impacts the mean of the corresponding statistical distribution of these measurements, but also by its width. Local lateral and axial blood flow components broaden the distributions (cf. Eq. 5). Bouwens et al. [44] quantified this effect in detail and derived rela- sels at two different time points at $535 \mathrm{~ms}$ (upper) and $1340 \mathrm{~ms}$ (lower). It is possible to differentiate between fast and slow flows, and to observe cardiac cycle pulsation over time. Reprinted from Spahr et al. [43], (C) Georg Thieme Verlag KG

tions between the mean and the variance of the Doppler frequency distribution for different modes of the illumination beam and detected light for low to high numerical apertures. For ophthalmic systems, Gaussian beam profiles and low numerical apertures can be assumed. The mean of the frequency distribution is then expressed by Eq. (3) (with $v_{z}=V \cos \alpha$ ) and the variance is given as:

$$
\sigma^{2}=\frac{n^{2} k_{0}^{2} w_{0}^{2}}{4 \pi^{2} f^{2}} v_{T}^{2}+\frac{1}{\pi^{2} l_{c}^{2}} v_{z}^{2} .
$$

In Eq. (6) $k_{0}$ is the central wave number, $v_{T}^{2}=v_{x}^{2}+v_{y}^{2}, w_{0}$ is the beam diameter at the entrance pupil, and $f$ is the focal length. The parameter $l_{c}=1 /\left(n k_{\sigma}\right)$ is the coherence length with the spectral bandwidth $k_{\sigma}$. It can be appreciated from Eq. (6) that the Doppler frequency bandwidth will be broadened by the influence of lateral as well as axial motion [44, 45].

The above mentioned characteristics made this technique easier to use in situations where samples can be fixed, e.g. as in optical coherence microscopy, but the suitability for retinal measurements has so far not been established. The first demonstrations to determine axial and lateral velocities were done in phantom measurements (Fig. 7.7, here only lateral velocities shown). A 


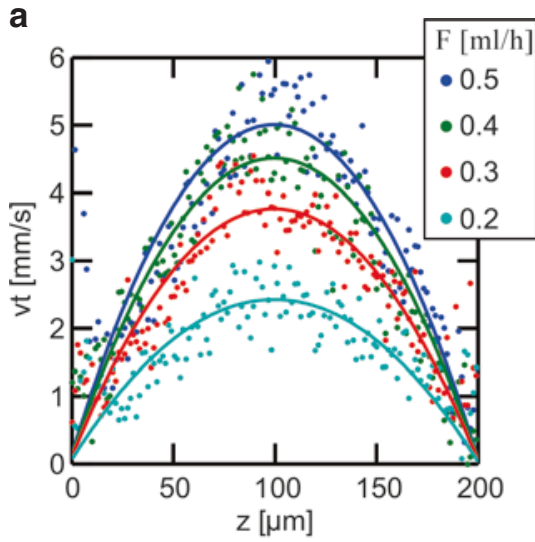

Fig. 7.7 Depth profiles at the center of a capillary $\left(\alpha=81^{\circ}\right)$ measured from the Doppler frequency bandwidth with extended focus OCM (xfOCM). (a) The lateral flow component measured for different flow rates. Parabolae are fitted to the measurements, assuming $V=0$ at the capillary wall. (b) The maxima of the parabolae are

glass capillary tube was connected to a syringe pump. To mimic the properties of a scattering fluid a solution of polystyrene beads was pumped through the capillary with a constant flow rate. Parabolic profiles were fitted to the measured velocities (a) and the derived maximal velocities were plotted against set velocities by the syringe pump (b). The results show a close representation of the expected values.

\subsubsection{Amplitude Based Flow Quantification}

Conventional Doppler OCT techniques as discussed above rely on the OCT signal phase for flow velocity quantification. These methods have proven accurate under the conditions that the Doppler angle is known and significantly away from perpendicularity, and that the lateral component is small enough to enable meaningful phase differences to be measured. It can, however, be challenging to fulfill these requirements in vivo, not only because the OCT system has to be configured to achieve phase stability, but most importantly because flow velocities in retinal vessels away from the optic nerve are dominated by the lateral velocity component. Amplitude-based b

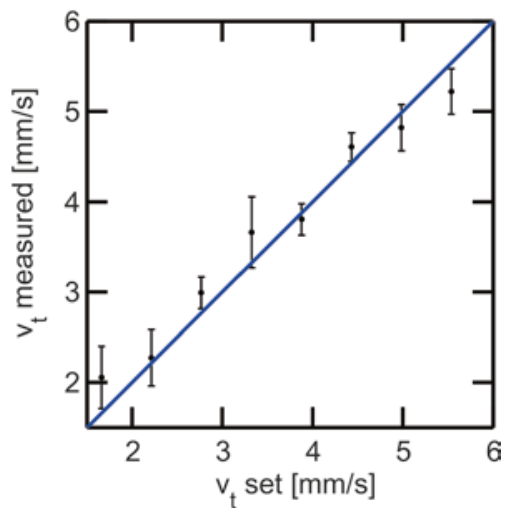

extracted and compared with the expected velocity as set by the syringe pump under laminar flow conditions. Error bars represent standard deviation over ten measurements. Reprinted from Bouwens et al. [44], with permission of The Optical Society (OSA)

methods try to address these drawbacks by analyzing the fluctuations of the OCT signal in response to the finite transit time of the scattering particles as the flow passes through the illumination beam.

OCT is a coherent technique and as such it is inherently subject to speckle. This phenomenon arises from the coherent superposition of light scattered from multiple points within a sample in a similar fashion to speckle observed in ultrasound images. The OCT signal at a given location in the sample is a complex value $F$ (the OCT complex amplitude), and is the result of the superposition of phasors due to the signal from each scattering particle inside the OCT resolution volume. Assuming a random distribution of scatterers, the statistics of the speckle amplitude are described by a random process. As particles move due to their flow velocity, a subset of scatterers moves out of the OCT probing volume, a new subset moves into it and a fraction remains. This continuous process produces a change in the coherent phasor sum as a function of time, and therefore the complex value of the tomogram at a given location.

The squared magnitude of the phasor sum is the OCT intensity $I$, which is generally displayed on a logarithmic (or another nonlinear) scale to show the structural OCT image. The intensity fluctuations also follow specific statistics linked 
to the fluctuations of $F$, and carry information about particle flow as well. The square root of $I$, the OCT amplitude, also presents a similar relationship. For this reason we refer to all these techniques as amplitude-based velocimetry.

In general, the faster the particles are replaced inside the OCT probing volume due to their flow speed, the faster the complex amplitude and intensity signals will fluctuate. Amplitude-based velocimetry links the dynamics of these fluctuations to the flow velocity, as we will see in the following sections. It is important to note that amplitude velocimetry techniques in OCT are less mature than phase-based methods, in part due to challenges in the correct interpretation and analysis of the data, as well as due to different, although potentially simpler, implementation at the hardware level. We start this section considering the statistics of the fluctuations of the complex amplitude, and later we continue with focusing on intensity fluctuations.

\subsubsection{Complex Amplitude: Dynamic Light Scattering Optical Coherence Tomography}

Dynamic light scattering optical coherence tomography (DLS-OCT) provides a comprehensive model for the OCT complex-valued signal and its temporal evolution in the presence of moving scatterers, like red blood cells in vascular flow. DLS-OCT represents a well-validated link between the OCT signal and moving scatterers in a single scattering regime.

The first-order autocorrelation function of the complex-valued OCT signal is calculated as [46-50]

$$
g^{(1)}(\hat{z}, \tau)=\left\langle F(\hat{z}, \tau) F^{*}(\hat{z}, 0)\right\rangle,
$$

where $F$ represents the complex backscattering signal of the sample at depth $\hat{z},\langle\ldots\rangle$ represents an ensemble average, and ${ }^{*}$ the complex conjugate. $F$ is given by the Fourier transform of the fringe signal [51]. We define the axial propagation direction of the beam as $z$, and the lateral plane dimensions as $x$ and $y$, and the flow velocity vector as $\left(v_{x}, v_{y}, v_{z}\right)$. The autocorrelation of a signal compares its amplitude at variable time differences $\tau$ and thus provides information on the typical time scale at which previous signal values still influence the present signal. Equation (7) is customarily normalized by a factor $\left.1 /<|F(\hat{z}, 0)|^{2}\right\rangle$, such that when the signal is perfectly correlated $g^{(1)}=1$, and when the signal is totally decorrelated $g^{(l)}=0$. The normalized $g^{(l)}$ is approximately given by [51]

$$
\begin{gathered}
g^{(1)}(\tau)=\exp \left[-i F_{D}\left(v_{z}\right) \tau\right] \exp \left[-F_{B}(D) \tau\right] \exp \left[-F_{G}\left(\frac{\partial v_{z}}{\partial x}, \frac{\partial v_{z}}{\partial y}, \frac{\partial v_{z}}{\partial z}\right) \tau^{2}\right] \times \\
\exp \left[-F_{x}\left(v_{x}^{2}\right) \tau^{2}\right] \exp \left[-F_{y}\left(v_{\mathrm{y}}^{2}\right) \tau^{2}\right] \exp \left[-F_{z}\left(v_{z}^{2}\right) \tau^{2}\right]
\end{gathered}
$$

where all $F()$ functions are positive and define different contributions to the decay of the correlation of the signal with $\tau$ [50]. $F_{D}$ is related to the familiar Doppler term that has been discussed before in Eqs. (2) and (3) and is part of the only imaginary exponent, $F_{B}$ a Brownian motion term with diffusion constant $D, F_{G}$ a gradient term, and $F_{x}, F_{y}, F_{z}$ velocity terms [51]. Terms that contribute comparatively little to the decorrelation have been omitted here [50]. Under normal flow conditions, also the Brownian motion contribution can be ignored, and gradient and velocity terms dominate.
Figure 7.8a shows a representative $g^{(1)}(\tau)$ calculated from an OCT flow signal. The amplitude is shown in grey, exhibiting a Gaussian decay with $\tau$. If an isotropic resolution volume is used and the flow velocity is mostly perpendicular to the OCT beam, the amplitude decay is insensitive to the flow direction and directly related to the total flow speed. Gradient effects play a role when axial flow is present and destroy the direct relationship between decay and total flow speed. The phase, in purple, shows a linear decrease with $\tau$ (with phase wrapping effects), and its evolution is directly linked to the axial velocity of 
Fig. 7.8 (a) Exemplary DLS-OCT curve from an OCT signal. The amplitude (grey solid) and the phase (purple dashed) as a function of time difference. The upper pair represents a sample at moderate flow while the lower pair corresponds to fast flow. (b) The Fourier pair of $g^{(l)}(\tau)$ shows the Doppler peak as generally visualized in Doppler OCT. The location of the peak is given by the phase slope in $g^{(I)}(\tau)$, while its width is related to the $g^{(I)}(\tau)$ decay
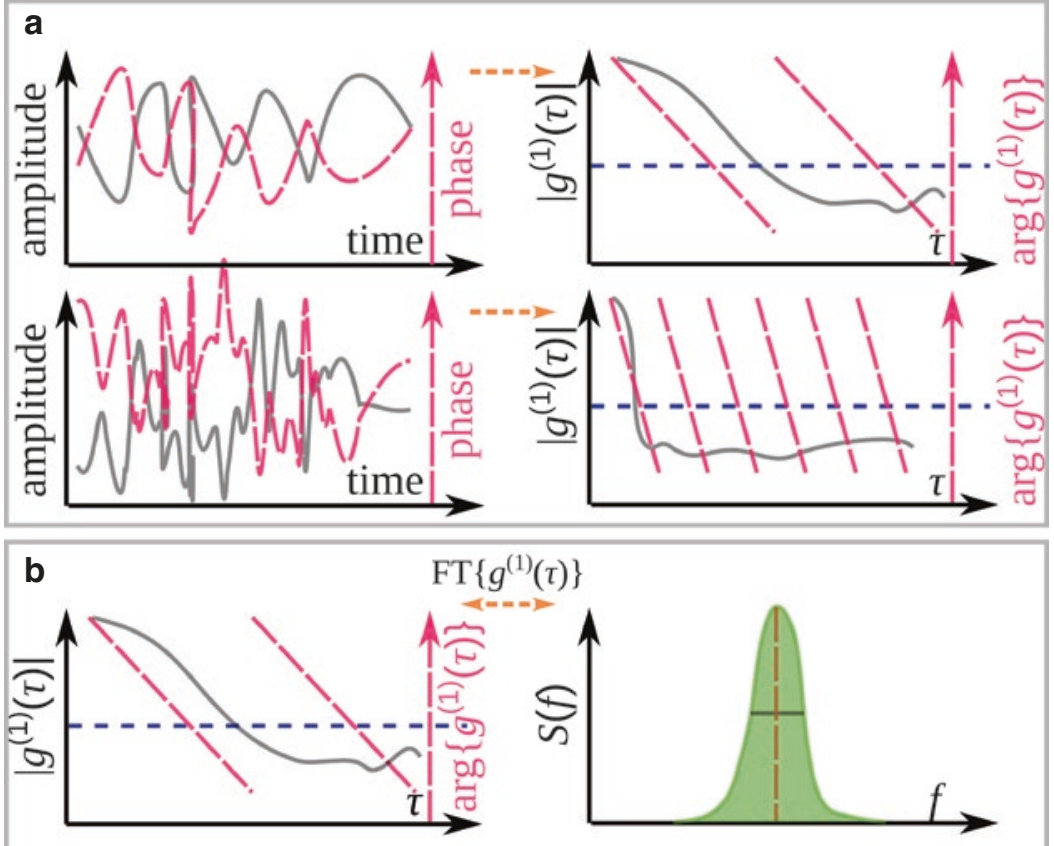

the scatterers. Not only the phase information is directional, but also a change in the sign of the axial velocity reverses the sign of the phase evolution.

$g^{(I)}(\tau)$ contains a wealth of information about scatterer motion and provides insights into the behavior of not only complex-amplitude velocimetry, but also phase methods. In Fig. 7.8b, inverse Fourier transformation of $g^{(1)}(\tau)$ provides the power spectrum of the OCT signal. The location of the peak (what is generally called the Doppler peak) is given by the phase slope in $g^{(I)}(\tau)$. The broadening of the peak, which is measured in Doppler variance, is dependent on all the contributions to the decorrelation in Eq. (8), including lateral flow and axial gradients. This explains the sensitivity of Doppler variance to lateral flow, and makes evident the need to also incorporate gradient effects into the Doppler variance framework for accurate velocimetry.

In practice, flow data is acquired by recording B-scans with a slow lateral scanning or by recording M-mode scans without lateral scanning at multiple locations. The complex-valued signal is then analyzed to calculate $g^{(I)}(\tau)$, which is either used in a fit to determine the decay coefficients in Eq. (8) or analyzed to find a decorrelation rate.
The decorrelation rate is then displayed in a twodimensional cross-sectional speckle decorrelation map (see Fig. 7.9a), with a corresponding structural image given by the intensity (see Fig. 7.9b). The decorrelation map can then be transformed into a flow speed map using Eq. (8) under many practical conditions.

\subsubsection{Intensity: Speckle Decorrelation}

Making use of the Siegert relationship, which states that under certain conditions $g^{(2)}=1+\left|g^{(1)}\right|^{2}[50]$, we can transform Eq. (8) into the second order autocorrelation function for the OCT signal intensity $I$ defined as,

$$
g^{(2)}(\tau)=\frac{\langle I(\tau) I(0)\rangle}{\langle I(\tau)\rangle\langle I(0)\rangle},
$$

which describes the so-called speckledecorrelation approach, also known as intensity-based DLS-OCT (iDLS-OCT). In this technique, only the fluctuations in intensity of the OCT signal (as shown in Fig. 7.9b) are analyzed using an autocorrelation approach, and the decorrelation rate is calculated. The main advantage of this technique is the fact that most 
a

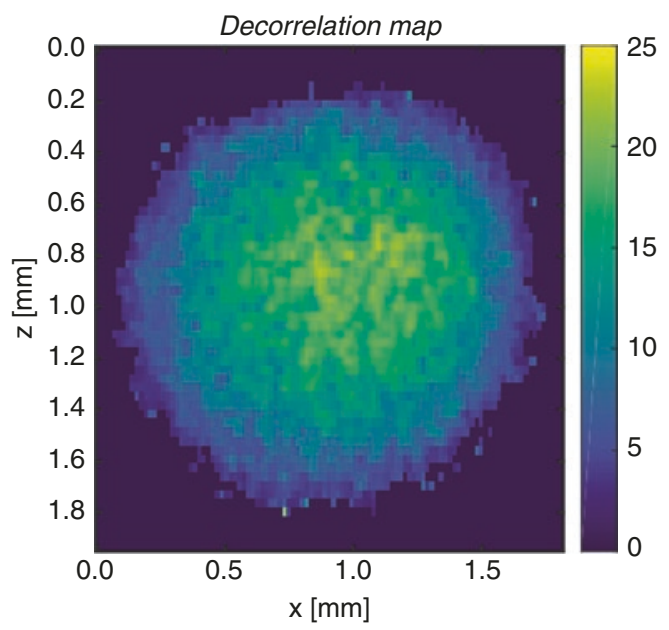

b

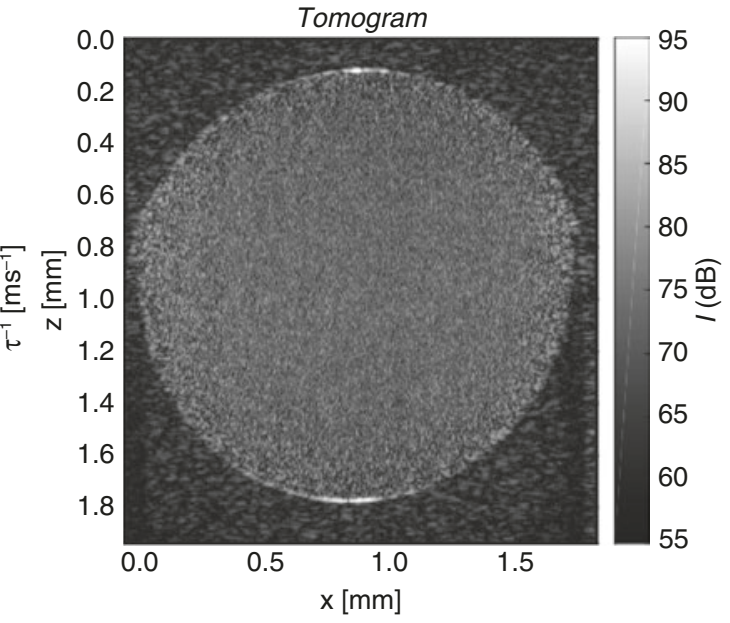

Fig. 7.9 Representative DLS-OCT measurement in a flow phantom setup. (a) Decorrelation analysis of the complexamplitude tomogram. (b) Intensity tomogram acquired by a slow lateral scan

clinical wavelength-swept source OCT systems are not phase stable, and therefore cannot be used for acquiring DLS-OCT data. In contrast, phase instability does not affect the decorrelation of the intensity signal, and therefore iDLSOCT data can be acquired with any OCT system. Similarly to the $g^{(l)}(\tau)$ decay, there is no directionality information in speckle decorrelation. With some transformations to account for Brownian motion and flow gradients, the decorrelation time is approximately inversely proportional to the flow speed when no significant axial component of the flow is present, which is precisely the case for retinal flow away from the optic nerve head $[52,53]$.

There are important practical considerations regarding the use of amplitude-based methods. Because the quantification lies in the decay of the autocorrelation function, such decay has to be properly sampled. This means that the temporal resolution of the OCT system, corresponding to the revisiting time of the scanning paradigm, has to resolve the decorrelation rate of the flow and other system parameters. If there is insufficient time sampling, we move into the realm of traditional OCT angiography techniques which do not sample the decay in case of flow, but only distinguish between moving particles and static tissue in an effectively binary way.
A different take on the amplitude decorrelation techniques consists of analyzing the properties of the members of the ensemble in Eqs. (8) and (9) instead of taking the ensemble average. This is in essence the principal component analysis (PCA) presented by Mohan et al. [54] Experiments on phantoms showed the potential for flow velocity quantification of this method with a reduced number of data points. In addition, analysis of in vivo blood flow in a mouse ear showed good agreement with conventional OCT Doppler measurements. These properties make this method attractive for quantitative retinal flow imaging, but further studies are needed to demonstrate its applicability.

DLS-OCT and its derivatives have significant potential to provide detailed information in retinal blood flow. However, most validations have been carried out in conditions that are far from those present in retinal flow, such as using solutions where single scattering is dominant, or using solid phantoms. Although there are publications with in vivo cerebral blood flow results, no in vivo retinal flow has been demonstrated yet. The most attractive property of DLS-OCT for ophthalmic imaging is the potential for blood flow quantification with a single imaging beam, without knowledge of the geometry or corrections for Doppler angle. 


\subsubsection{Alternative Speckle Decorrelation Methods}

In addition to the widely used speckle decorrelation method described above, several noteworthy alternative methods have been developed with potential clinical use. These methods sit between fully quantitative decorrelation methods and OCT angiography: their time sampling is typically too sparse to sample the decay of large retinal vessels, but sufficient to give a coarse degree of quantification for smaller vessels.

The split-spectrum amplitude decorrelation angiography (SSADA) algorithm [55] is similar to iDLS-OCT but uses the square root of the intensity signal and a modified normalization constant, and the axial resolution is reduced via spectrum splitting to perform averaging. Due to its relationship with the second-order autocorrelation function (Eq. 9), theoretically it has the potential for quantification of flow. However, all in vivo retinal measurements so far have had insufficient temporal resolution to obtain meaningful quantification. Tokayer et al. [56] showed that quantification could be achieved by increasing the time sampling by analyzing M-mode scans with a static beam, which were performed on a blood infused capillary sample.

The assessment of OCT intensity decorrelation at different time intervals also forms the basis for the variable interscan time analysis (VISTA) method of Choi et al. [57]. In this method OCTA intensity decorrelation images are obtained at two time intervals with the longer interval $(3.0 \mathrm{~ms})$ at double the rescan time of the short interval $(1.5 \mathrm{~ms})$. This is in essence an autocorrelation function with only two sampled time differences $\tau$. This limited information is used to determine fast flows (those decorrelated at both time intervals) and a range of slow flows (those with different degree of decorrelation at both time intervals). This range is mapped to a color scale which provides information to qualitatively assess flow velocity differences between (capillary) vessels [58]. An example of VISTA flow imaging is shown in Fig. 7.10 for a patient with polypoidal choroidal vasculopathy (PCV). Figure $7.10 \mathrm{a}$, d show ICG images where the polyp could be clearly visualized with the bright periphery of the polyp indicating increased flow towards the polyp wall (arrow). The branching vascular network (BVN, marked by the dashed line) is visible but obscured by the fluorescence of vessels at different axial depths. In OCTA projections from different depths (Fig. 7.10b, e) it can be appreciated that the polyp is located in a more superficial layer than the choroidal neovascularization. Figure 7.10c, f show VISTA images from the same depth as the OCTA projections. In Fig. 7.10b as well as Fig. 7.10c, it can be seen that blood flow in the outer part of the polyp is faster compared to its central part, which is in good agreement with the ICGA finding (inset Fig. 7.10d). The BVN (see inset Fig. 7.10f) presents relative slow flow compared to the regular retinal vessels. Although VISTA images could be a good qualitative aid in assessing retinal flow properties, the unknown temporal relation of the VISTA decorrelation signals and their limited time sampling currently limits the extension of this method into a full quantitative flow measurement.

\subsection{Discussion and Conclusion}

Noninvasive quantitative blood flow measurements in the human eye hold the potential to provide new clinically relevant insights into retinal hemodynamics in health and disease. For its future integration into clinical practice, however, a robust, fast, and reliable measurement will be required. Typical currently employed approaches for OCT-based velocimetry rely on analyzing modulations in either or both of the phase and amplitude of the complex OCT signal that is backscattered from moving red blood cells. While recent research yielded significant progress in each individual approach, all currently still also hold limitations and difficulties to be overcome in the future. Therefore, any of the methods may be superior in certain application scenarios and for imaging different locations of the retina, and a combination of different methods may be most feasible for providing a truly versatile clinical measurement technique. 

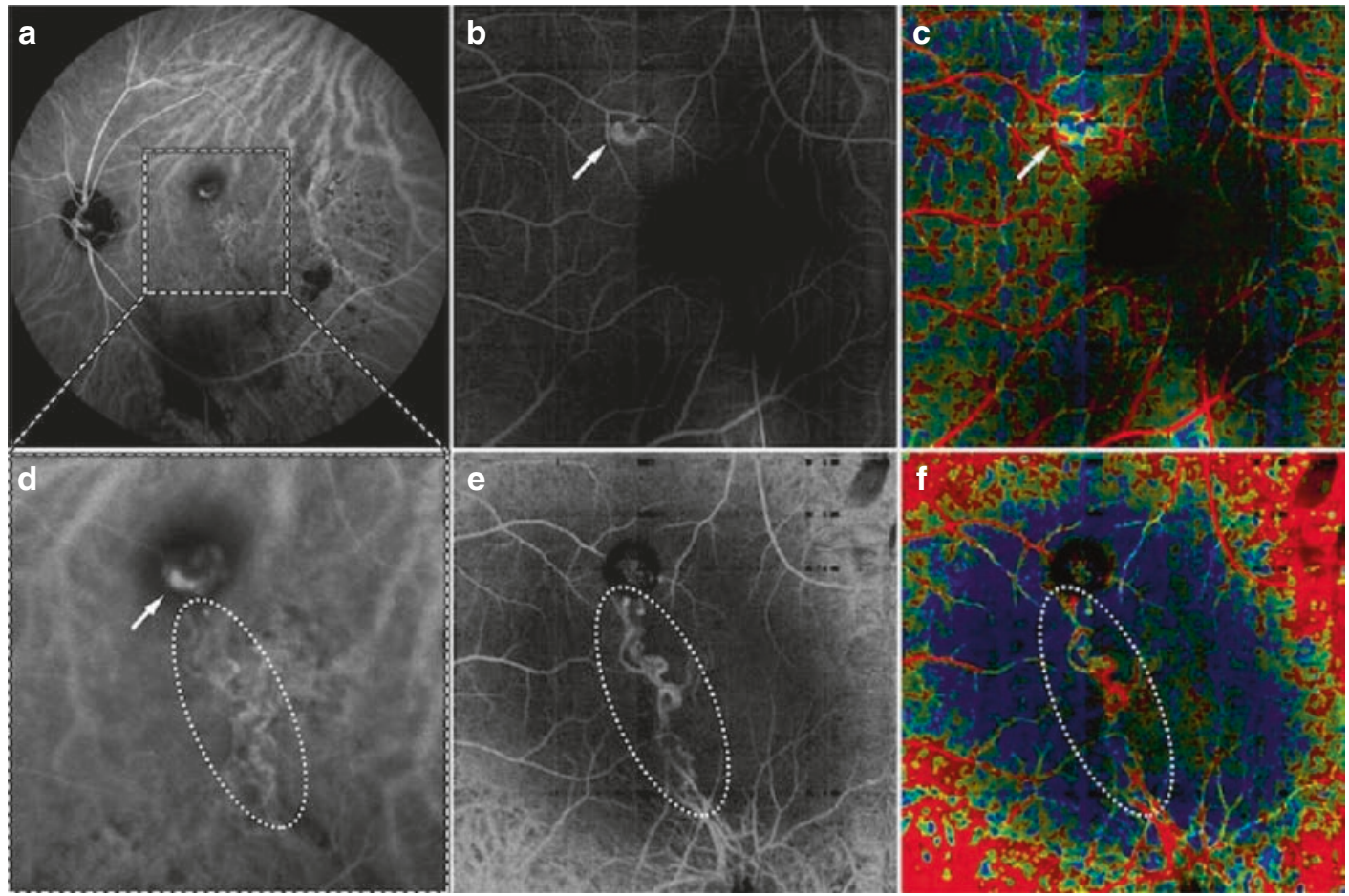

Fig. 7.10 Figure adopted from Rebhun et al. [58, 59]. Polypoidal choroidal vasculopathy (PCV) on VISTAOCTA; red indicates faster blood flow speeds, and blue indicates slower speeds. Left eye of a 61-year-old woman with PCV. (a) ICGA showing the branching vascular network (BVN) and polypoidal lesion. (d) Larger scale of the macular area documented by ICGA in (a) (white dashed line), with the BVN (white dotted line) and a polyp with

Current clinically employed OCT technology does not yet offer the required temporal repetition rate and overall acquisition speed for repeated scans over extended retinal regions that would allow feasible quantitative velocimetry over comparable field of views that are typically employed in structural OCT and qualitative OCTA measurements. Independent of the specific analysis approach, the same moving blood cell has to be imaged repeatedly over multiple measurements, which dictates the minimum scan repetition rate as well as the minimum number of repeated scans at an individual vessel location for fastest and slowest flow, respectively. The physiological change in flow rate across the heart cycle bright periphery toward the polyp wall and dark center (white arrow). (b, c, e, f) OCTA images of the same eye, but projected from different axial depths (different segmentation levels) capturing different components of the lesion. (b, c) Clearly shows the polyp with blood flow toward the polyp wall, but not in the center. (e, f) Clearly shows the BVN with relatively fast flow (see f). (c, f) are OCTA scans applying VISTA-OCTA

further complicates the situation and may additionally require imaging of individual vessels over more than a second to provide a comprehensive signal. Nevertheless, localized measurements of flow within a number of individually selected vessels, for instance around the optic nerve head, may already provide clinically useful information on current clinical device hardware. Future generations of OCT devices will certainly help mitigating these bottlenecks. Ultimately, the combination of quantitative retinal blood flow with oximetry measurements may contribute another important puzzle piece for the comprehensive understanding of the role of dysfunctional metabolic supply in retinal disease. 


\section{References}

1. Harris A, Chung HS, Ciulla TA, Kagemann L. Progress in measurement of ocular blood flow and relevance to our understanding of glaucoma and agerelated macular degeneration. Prog Retin Eye Res. 1999;18(5):669-87.

2. Harris A, Kagemann L, Ehrlich R, Rospigliosi C, Moore D, Siesky B. Measuring and interpreting ocular blood flow and metabolism in glaucoma. Can J Ophthalmol. 2008;43(3):328-36.

3. Flammer J, Orgül S, Costa VP, Orzalesi N, Krieglstein GK, Serra LM, Renard J-P, Stefánsson E. The impact of ocular blood flow in glaucoma. Prog Retin Eye Res. 2002;21(4):359-93.

4. Nakazawa T. Ocular blood flow and influencing factors for glaucoma. Asia Pac J Ophthalmol (Phila). 2016;5(1):38-44.

5. Schmetterer L, Wolzt M. Ocular blood flow and associated functional deviations in diabetic retinopathy. Diabetologia. 1999;42(4):387-405.

6. Ting DS, Cheung GC, Wong TY. Diabetic retinopathy: global prevalence, major risk factors, screening practices and public health challenges: a review. Clin Experiment Ophthalmol. 2016;44(4):260-77.

7. Pournaras CJ, Riva CE. Retinal blood flow evaluation. Ophthalmologica. 2013;229(2):61-74.

8. Patel RC, Gao SS, Zhang M, Alabduljalil T, Al-Qahtani A, Weleber RG, Yang P, Jia Y, Huang D, Pennesi ME. Optical coherence tomography angiography of choroidal neovascularization in four inherited retinal dystrophies. Retina. 2016;36(12):2339-47.

9. Novais EA, Adhi M, Moult EM, Louzada RN, Cole ED, Husvogt L, Lee B, Dang S, Regatieri CV, Witkin AJ, Baumal CR, Hornegger J, Jayaraman V, Fujimoto JG, Duker JS, Waheed NK. Choroidal neovascularization analyzed on ultrahigh-speed swept-source optical coherence tomography angiography compared to spectral-domain optical coherence tomography angiography. Am J Ophthalmol. 2016;164:80-8.

10. Coscas F, Glacet-Bernard A, Miere A, Caillaux V, Uzzan J, Lupidi M, Coscas G, Souied EH. Optical coherence tomography angiography in retinal vein occlusion: evaluation of superficial and deep capillary plexa. Am J Ophthalmol. 2016;161:160-71 e1-2.

11. Chu Z, Lin J, Gao C, Xin C, Zhang Q, Chen CL, Roisman L, Gregori G, Rosenfeld PJ, Wang RK. Quantitative assessment of the retinal microvasculature using optical coherence tomography angiography. J Biomed Opt. 2016;21(6):66008.

12. Jia Y, Bailey ST, Hwang TS, McClintic SM, Gao SS, Pennesi ME, Flaxel CJ, Lauer AK, Wilson DJ, Hornegger J, Fujimoto JG, Huang D. Quantitative optical coherence tomography angiography of vascular abnormalities in the living human eye. Proc Natl Acad Sci U S A. 2015;112(18):E2395-402.
13. Zhang A, Zhang Q, Chen CL, Wang RK. Methods and algorithms for optical coherence tomography-based angiography: a review and comparison. J Biomed Opt. 2015;20(10):100901.

14. Stieger K, Munk MR, Giannakaki-Zimmermann H, Berger L, Huf W, Ebneter A, Wolf S, Zinkernagel MS. OCT-angiography: a qualitative and quantitative comparison of 4 OCT-A devices. PLoS One. 2017;12(5):e0177059.

15. Braaf, B. Angiography and polarimetry of the posterior eye with functional optical coherence tomography. 2015.

16. Pournaras CJ, Rungger-Brandle E, Riva CE, Hardarson SH, Stefansson E. Regulation of retinal blood flow in health and disease. Prog Retin Eye Res. 2008;27(3):284-330.

17. Zinser G. Scanning laser Doppler flowmetry: principle and technique. Current concepts on ocular blood flow in glaucoma. The Hague: Kugler Publications; 1999. p. 197-204.

18. Riva C, Ross B, Benedek GB. Laser Doppler measurements of blood flow in capillary tubes and retinal arteries. Invest Ophthalmol Vis Sci. 1972;11(11):936-44.

19. Preussner P, Richard G, Darrelmann O, Weber J, Kreissig I. Quantitative measurement of retinal blood flow in human beings by application of digital image-processing methods to television fluorescein angiograms. Graefes Arch Clin Exp Ophthalmol. 1983;221(3):110-2.

20. Dervenis P, Dervenis N, Mikropoulou AM. Imaging modalities for assessing ocular hemodynamics. Exp Rev Ophthalmol. 2018;13(2):65-73.

21. Wang RK, Chu Z, Zhang Q, Wei W. Physical explanation and experimental demonstration of suspended scattering particles in motion creating non-vascular signal in OCT angiography. Invest Ophthalmol Vis Sci. 2018;59(9):3926.

22. Zhong Z, Petrig BL, Qi X, Burns SA. In vivo measurement of erythrocyte velocity and retinal blood flow using adaptive optics scanning laser ophthalmoscopy. Opt Express. 2008;16(17):12746-56.

23. Saeedi O, Tracey B, Renner C, Li J, Shah K, Tsai J, Chang L, Ou M. Determination of absolute erythrocyte velocity and flow in the human retinal microvasculature by direct visualization of ICG-labelled erythrocytes. Invest Ophthalmol Vis Sci. 2018;59(9):3950.

24. Chen Z, Milner TE, Dave D, Nelson JS. Optical Doppler tomographic imaging of fluid flow velocity in highly scattering media. Opt Lett. 1997;22(1):64.

25. Zhao Y, Chen Z, Saxer C, Xiang S, de Boer JF, Nelson JS. Phase-resolved optical coherence tomography and optical Doppler tomography for imaging blood flow in human skin with fast scanning speed and high velocity sensitivity. Opt Lett. 2000;25(2):114.

26. Wang XJ, Milner TE, Nelson JS. Characterization of fluid flow velocity by optical Doppler tomography. Opt Lett. 1995;20(11):1337. 
27. Leitgeb R, Schmetterer LF, Wojtkowski M, Hitzenberger CK, Sticker M, Fercher AF. In Flow velocity measurements by frequency domain short coherence interferometry. In: International Symposium on Biomedical Optics. Bellingham WA: SPIE; 2002. p 6.

28. White BR, Pierce MC, Nassif N, Cense B, Park BH, Tearney GJ, Bouma BE, Chen TC, de Boer JF. In vivo dynamic human retinal blood flow imaging using ultra-high-speed spectral domain optical Doppler tomography. Opt Express. 2003;11(25):3490-7.

29. Wang L, Xu W, Bachman M, Li GP, Chen ZP. Phaseresolved optical Doppler tomography for imaging flow dynamics in microfluidic channels. Appl Phys Lett. 2004;85(10):1855-7.

30. Zhang J, Chen ZP. In vivo blood flow imaging by a swept laser source based Fourier domain optical Doppler tomography. Opt Express. 2005;13(19):7449-57.

31. Hendargo HC, Zhao MT, Shepherd N, Izatt JA. Synthetic wavelength based phase unwrapping in spectral domain optical coherence tomography. Opt Express. 2009;17(7):5039-51.

32. Xia SY, Huang Y, Peng SZ, Wu YF, Tan XD. Robust phase unwrapping for phase images in Fourier domain Doppler optical coherence tomography. J Biomed Opt. 2017;22(3):36014.

33. Park BH, Pierce MC, Cense B, Yun SH, Mujat M, Tearney GJ, Bouma BE, de Boer JF. Real-time fiber-based multi-functional spectral-domain optical coherence tomography at $1.3 \mathrm{mu}$ m. Opt Express. 2005;13(11):3931-44.

34. Braaf B, Vermeer KA, Vienola KV, de Boer JF. Angiography of the retina and the choroid with phase-resolved OCT using interval-optimized backstitched B-scans. Opt Express. 2012;20(18):20516-34.

35. Wang YM, Bower BA, Izatt JA, Tan O, Huang D. Retinal blood flow measurement by circumpapillary Fourier domain Doppler optical coherence tomography. J Biomed Opt. 2008;13(6):064003.

36. Srinivasan VJ, Sakadzic S, Gorczynska I, Ruvinskaya $\mathrm{S}, \mathrm{Wu}$ W, Fujimoto JG, Boas DA. Quantitative cerebral blood flow with optical coherence tomography. Opt Express. 2010;18(3):2477-94

37. Haindl R, Trasischker W, Wartak A, Baumann B, Pircher M, Hitzenberger CK. Total retinal blood flow measurement by three beam Doppler optical coherence tomography. Biomed Opt Express. 2016;7(2):287-301.

38. Goodman JW. Introduction to Fourier optics. 2nd ed. New York, NY: McGraw-Hill; 1996. p. xviii, 441 p.

39. Hillmann D, Spahr H, Hain C, Sudkamp H, Franke G, Pfaffle C, Winter C, Huttmann G. Aberration-free volumetric high-speed imaging of in vivo retina. Sci Rep. 2016;6:35209.

40. Spahr H, Pfäffle C, Koch P, Sudkamp H, Hüttmann G, Hillmann D. Interferometric detection of 3D motion using computational subapertures in optical coherence tomography. Opt Express. 2018;26(15):18803-16.
41. Karamata B, Laubscher M, Leutenegger M, Bourquin S, Lasser T, Lambelet P. Multiple scattering in optical coherence tomography. I. Investigation and modeling. J Opt Soc Am A. 2005;22(7):1369-79.

42. Karamata B, Leutenegger M, Laubscher M, Bourquin $\mathrm{S}$, Lasser T, Lambelet P. Multiple scattering in optical coherence tomography. II. Experimental and theoretical investigation of cross talk in wide-field optical coherence tomography. J Opt Soc Am A. 2005;22(7):1380-8.

43. Spahr H, Hillmann D, Hain C, Pfaffle C, Sudkamp H, Franke G, Koch P, Huttmann G. [Imaging blood flow and pulsation of retinal vessels with full-field swept-source OCT]. Klin Monbl Augenheilkd 2016;233(12):1324-1330.

44. Bouwens A, Szlag D, Szkulmowski M, Bolmont T, Wojtkowski M, Lasser T. Quantitative lateral and axial flow imaging with optical coherence microscopy and tomography. Opt Express. 2013;21(15):17711-29.

45. Srinivasan VJ, Radhakrishnan H, Lo EH, Mandeville ET, Jiang JY, Barry S, Cable AE. OCT methods for capillary velocimetry. Biomed Opt Express. 2012;3(3):612-29.

46. Joo C, de Boer JF. Field-based dynamic light scattering microscopy: theory and numerical analysis. Appl Optics. 2013;52(31):7618-28.

47. Weiss N, van Leeuwen TG, Kalkman J. Localized measurement of longitudinal and transverse flow velocities in colloidal suspensions using optical coherence tomography. Phys Rev E Stat Nonlin Soft Matter Phys. 2013;88(4):042312.

48. Lee J, Wu W, Jiang JY, Zhu B, Boas DA. Dynamic light scattering optical coherence tomography. Opt Express. 2012;20(20):22262-77.

49. Huang BK, Choma MA. Resolving directional ambiguity in dynamic light scattering-based transverse motion velocimetry in optical coherence tomography. Opt Lett. 2014;39(3):521-4.

50. Uribe-Patarroyo N, Bouma BE. Velocity gradients in spatially resolved laser Doppler flowmetry and dynamic light scattering with confocal and coherence gating. Phys Rev E. 2016;94(2-1):022604.

51. Yun SH, Tearney GJ, de Boer JF, Bouma BE. Motion artifacts in optical coherence tomography with frequency-domain ranging. Opt Express. 2004;12(13):2977.

52. Uribe-Patarroyo N, Villiger M, Bouma BE. Quantitative technique for robust and noisetolerant speed measurements based on speckle decorrelation in optical coherence tomography. Opt Express. 2014;22(20):24411.

53. Uribe-Patarroyo N, Bouma BE. Rotational distortion correction in endoscopic optical coherence tomography based on speckle decorrelation. Opt Lett. 2015;40(23):5518-21.

54. Mohan N, Vakoc B. Principal-component-analysisbased estimation of blood flow velocities using optical coherence tomography intensity signals. Opt Lett. 2011;36(11):2068-70. 
55. Jia Y, Tan O, Tokayer J, Potsaid B, Wang Y, Liu JJ, Kraus MF, Subhash H, Fujimoto JG, Hornegger J, Huang D. Split-spectrum amplitude-decorrelation angiography with optical coherence tomography. Opt Express. 2012;20(4):4710-25.

56. Tokayer J, Jia Y, Dhalla AH, Huang D. Blood flow velocity quantification using split-spectrum amplitude-decorrelation angiography with optical coherence tomography. Biomed Opt Express. 2013;4(10):1909-24.

57. Choi W, Moult EM, Waheed NK, Adhi M, Lee B, Lu CD, de Carlo TE, Jayaraman V, Rosenfeld PJ, Duker JS, Fujimoto JG. Ultrahigh-speed, swept-source optical coherence tomography angiography in nonexudative age-related macular degeneration with geographic atrophy. Ophthalmology. 2015;122(12):2532-44.
58. Ploner SB, Moult EM, Choi W, Waheed NK, Lee B, Novais EA, Cole ED, Potsaid B, Husvogt L, Schottenhamml J, Maier A, Rosenfeld PJ, Duker JS, Hornegger J, Fujimoto JG. Toward quantitative optical coherence tomography angiography: visualizing blood flow speeds in ocular pathology using variable interscan time analysis. Retina. 2016;36(Suppl 1):S118-26.

59. Rebhun CB, Moult EM, Novais EA, Moreira-Neto C, Ploner SB, Louzada RN, Lee B, Baumal CR, Fujimoto JG, Duker JS, Waheed NK, Ferrara D. Polypoidal choroidal vasculopathy on swept-source optical coherence tomography angiography with variable interscan time analysis. Transl Vis Sci Technol. 2017;6(6):4.

Open Access This chapter is licensed under the terms of the Creative Commons Attribution 4.0 International License (http://creativecommons.org/licenses/by/4.0/), which permits use, sharing, adaptation, distribution and reproduction in any medium or format, as long as you give appropriate credit to the original author(s) and the source, provide a link to the Creative Commons license and indicate if changes were made.

The images or other third party material in this chapter are included in the chapter's Creative Commons license, unless indicated otherwise in a credit line to the material. If material is not included in the chapter's Creative Commons license and your intended use is not permitted by statutory regulation or exceeds the permitted use, you will need to obtain permission directly from the copyright holder. 